\section{Effects of Refractive Index and Diffuse or Specular Boundaries on a Radiating Isothermal Layer}

\section{R. Siegel ${ }^{1,3}$ and C. M. Spuckler ${ }^{2,3}$}

\section{Nomenclature}

$a=$ absorption coefficient of layer, $\mathrm{m}^{-1}$

$c=$ specific heat of solid, $\mathrm{W} \cdot \mathrm{s} / \mathrm{kg} \cdot \mathrm{K}$

$D=$ thickness of plane layer, $\mathrm{m}$

$e_{\lambda b}=$ blackbody spectral emissive power, $\mathrm{W} / \mathrm{m}^{2} \cdot \mu \mathrm{m}$

$E_{3}=$ exponential integral function, $E_{3}(\xi)=\int_{0}^{1} \mu \exp$ $(-\xi / \mu) d \mu$

$F_{m}=$ blackbody fraction of $m$ th spectral band

$n=$ index of refraction

$q=$ heat flux, $\mathrm{W} / \mathrm{m}^{2} ; q_{\lambda}$, spectral flux, $\mathrm{W} / \mathrm{m}^{2} \cdot \mu \mathrm{m}$

$q_{\lambda 1}, q_{\lambda 2}=$ externally incident spectral radiation fluxes, $\mathrm{W} / \mathrm{m}^{2} \cdot \mu \mathrm{m}$

$T_{e}=$ temperature of environment, $\mathrm{K} ; \tilde{T}_{e}=T_{e} / T_{0}$

$T_{0}=$ layer temperature at time $t=0, \mathrm{~K}$

$T_{w}=$ absolute temperature of layer, $\mathrm{K} ; T_{w}=T_{w} / T_{0}$

$T_{1}, T_{2}=$ temperatures of radiating surroundings, $\mathrm{K}$

$t=$ time, s; dimensionless time, $t=t \sigma T_{0}^{3} / \rho c D$

$\alpha, \epsilon=$ absorptance and emittance of plane layer

$\theta=$ angle of incidence

$\rho=$ reflectivity of interface; density of solid, $\mathrm{kg} / \mathrm{m}^{3}$

$\sigma=$ Stefan-Boltzmann constant, $\mathrm{W} / \mathrm{m}^{2} \cdot \mathrm{K}^{4}$

$\tau=$ transmittance of path at angle $\chi, \tau=\exp$ $(-a D / \cos x)$

$x=$ angle of refraction

Subscripts

dif $=$ for diffuse surfaces

$i, 0=$ incoming and outgoing radiation

$m=$ the $m$ th spectral band

$s=$ for specular surfaces

$\lambda=$ spectral quantity

$\perp, \boldsymbol{H}=$ components for perpendicular and parallel polarization

\section{Superscripts}

$i, 0=$ at inside or outside surface of an interface

\section{Introduction}

Ceramics are under development for high-temperature applications such as in combustion chambers of high-speed aircraft. Some ceramics are reinforced with ceramic fibers, and to protect the composite from the combustion environment it is coated with another ceramic to form a laminated layer. To model these materials thermally in order to study possible radiation effects arising from their partial transparency, it is necessary to specify the nature of reflections at their interfaces. $A$ convenient assumption is that reflections are diffuse; this

\footnotetext{
'Senior Research Scientist: Fellow ASME.

2Research Scientist; Assoc. Mem. ASME.

'NASA Lewis Research Center, 21000 Brookpark Rd., Cleveland, OH 44135.

Contributed by the Heat Transfer Division of THE AmERICAN SOCIETY of Mechanical Engineers. Manuscript received by the Heat Transter Division June 1993; revision received November 1993. Keywords: Radiation. Associate Technical Editor: M. F. Modest.
}

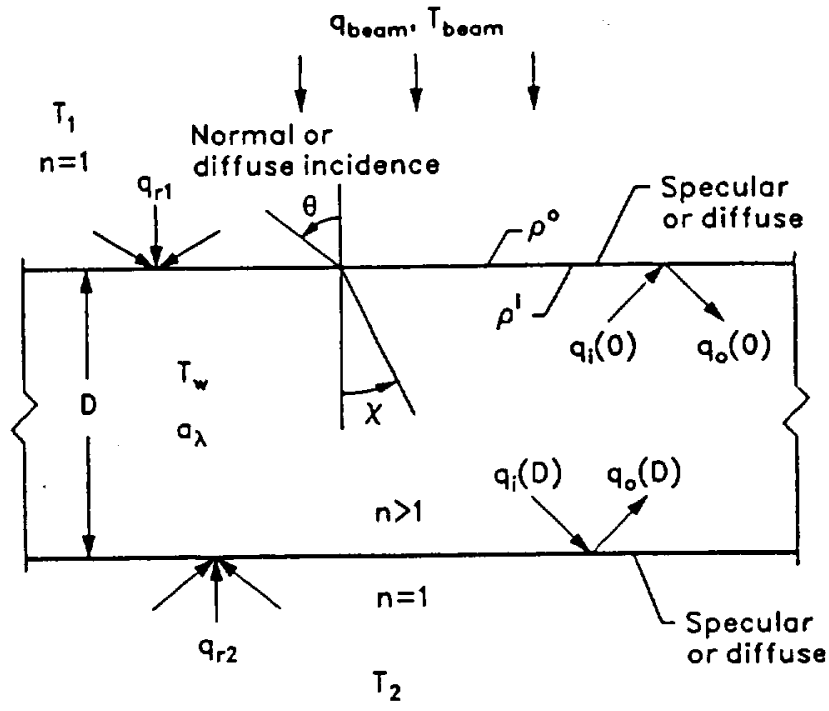

Fig. 1 Plane layer with specular or diffuse boundaries and with dilfuse or normally Incident radiation

may be realistic because some external surfaces and internal interfaces of ceramics are rough. Some surfaces, however, may be smooth so they are specularly reflecting. This note will examine in a simple way by considering an isothermal layer, some effects of diffuse versus specular boundary conditions. In some cases the diffuse condition yields increased temperatures due to augmented retention of incident energy by internal reflections. This is a function of the refractive index of the material. Much of the work in the literature is for glass, $n=1.5$; for ceramics $n$ can be significantly larger, thus providing increased internal radiation effects. The limiting condition is examined where there is no scattering inside the layer as this demonstrates the maximum effect of the surface condition. For specular surfaces, a small amount of scattering within the layer produces a partially diffuse behavior; for large scattering, diffuse behavior will result.

\section{Analysis}

Absorptance of Layer With Specular Boundaries and No Internal Scattering. The layer in Fig. 1 with refractive index $n>1$ has incident diffuse or beam energy. The layer partially absorb's radiation, but does not scatter internally. For specularly reflecting surfaces, radiation in each incident direction $\theta$ is reiracted and internally reflected as given by Fresnel relations. The fraction of energy absorbed is given as (Siegel and Howell, 1992, p. 929): $\alpha(\theta)=\left[1-\rho^{\circ}(\theta)\right][1-\tau(\theta)] /[1-$ $\left.\rho^{0}(\theta) \tau(\theta)\right]$ where the internal transmittance is $\tau(\theta)=\exp [-a D /$ $\left.\rho^{\circ}(\theta) \tau(\theta)\right]$ where the ingle of refraction is $\chi(\theta)=\sin ^{-1}[(\sin \theta) /$ $n$ ]. The reflectivity $\rho^{\circ}(\theta)$ is a function of the polarization component so $\alpha(\theta)$ has values corresponding to the perpendicular and parallel components. For diffuse incidence of unpolarized radiation, the absorptance of a layer with specular surfaces is found by integrating the absorbed energy for all incident solid angles to give

$$
\alpha_{s}(n, a D)=\int_{0}^{\pi / 2}\left[\alpha_{\perp}(\theta)+\alpha_{1}(\theta)\right] \cos \theta \sin \theta d \theta
$$

where the $\rho^{\circ}$ needed to obtain $\alpha_{1}$ and $\alpha_{1}$ are

$$
\begin{aligned}
& \rho_{\perp}^{0}(\theta)=\left[\frac{\left(n^{2}-\sin ^{2} \theta\right)^{1 / 2}-\cos \theta}{\left(n^{2}-\sin ^{2} \theta\right)^{1 / 2}+\cos \theta}\right]^{2} \\
& \rho_{i}^{0}(\theta)=\left[\frac{n^{2} \cos \theta-\left(n^{2}-\sin ^{2} \theta\right)^{1 / 2}}{n^{2} \cos \theta+\left(n^{2}-\sin ^{2} \theta\right)^{1 / 2}}\right]^{2}
\end{aligned}
$$


$W$ ith all quantities in Eq. (1) in terms of $\theta$, the integration is performed numerically for various $n$ and $a D$. For the special case of normal incidence, $\alpha_{s}=\left(1-\rho^{0}\right)(1-\tau) /\left(1-\rho^{\circ} \tau\right)$ where $\rho^{\circ}=[(n-1) /(n+1)]^{2}$ and $\tau=\exp (-a D)$.

For emission from an isothermal layer, the local internal emission is considered for each direction. Radiation internally incident on a boundary is totally reflected if it arrives at an angle larger than the critical angle for reflection, which depends on $n$. The formulation by Gardon (1956) for the emittance of an isothermal layer with specular boundaries provides the same result as $\alpha_{s}$ in Eq. (1); this is expected from Kirchhoff's law.

Absorptance of a Layer With Diffuse Boundaries and No Internal Scattering. The layer boundaries are now considered diffuse. For a rough surface it is assumed that incident radiation will strike surface elements at random angles so the surface reflectivity is independent of the angular distribution of the incident energy. Hence all energy incident upon and leaving a boundary is considered to behave in a diffuse manner. The diffuse reflectivity is given by the hemispherically averaged relation of the Fresnel equations (Siegel and Howell, 1992, p. 115),

$$
\begin{aligned}
\rho_{\text {dir }}^{\circ}(n)= & \frac{1}{2}+\frac{(3 n+1)(n-1)}{6(n+1)^{2}}+\frac{n^{2}\left(n^{2}-1\right)^{2}}{\left(n^{2}+1\right)^{3}} \ln \left(\frac{n-1}{n+1}\right) \\
& -\frac{2 n^{3}\left(n^{2}+2 n-1\right)}{\left(n^{2}+1\right)\left(n^{4}-1\right)}+\frac{8 n^{4}\left(n^{4}+1\right)}{\left(n^{2}+1\right)\left(n^{4}-1\right)^{2}} \ln (n)
\end{aligned}
$$

The absorptance of the layer is obtained by considering its interaction with a unit incident flux, $q_{r 1}=1$. The outgoing flux from the internal side of each diffuse boundary (see Fig. $1)$ is then

$$
q_{0}(0)=1-\rho_{\mathrm{dif}}^{o}+q_{i}(0) \rho_{\mathrm{dif}}^{i} ; q_{0}(D)=q_{i}(D) \rho_{\mathrm{dif}}^{i} \quad(4 a, b)
$$

where $\rho_{\text {dif }}^{\prime}$ is at the internal side of a diffuse boundary. As a result of transmission within the layer, the fluxes at the two internal sides of the boundaries are related by (Siegel and Howell, 1992, p. 707)

$$
q_{i}(0)=2 q_{0}(D) E_{3}(a D) ; q_{i}(D)=2 q_{o}(0) E_{3}(a D)
$$

Equations (4) and (5) are solved for $q_{0}(0), q_{0}(D), q_{i}(0)$, and $q_{i}(D)$. The fraction of incident energy that is absorbed is $\alpha_{\mathrm{dif}}$ $=1-\left(1-\rho_{\mathrm{dif}}^{i}\right)\left[q_{i}(0)+q_{i}(D)\right]$, which yields

$$
\alpha_{\mathrm{dir}}(n, a D)=\left(1-\rho_{\mathrm{dir}}^{o}\right) \frac{1-2 E_{3}(a D)}{1-\rho_{\mathrm{dir}}^{i} 2 E_{3}(a D)}
$$

To place $\alpha_{\text {dir }}$ in terms of the reflectivity $\rho_{\text {dif }}^{o}$ on the outer surface of a boundary, the relation can be used that (Richmond, 1963) $\rho_{\text {dif }}^{i}=1-\left(1 / n^{2}\right)\left(1-\rho_{\text {dif }}^{o}\right)$.

Heat Balance Relations for Layer Temperature With Transfer Only by Radiation. The layer absorptances are spectral quantities since the absorption coefficient depends on wavelength. Two examples are given to demonstrate effects of diffuse and specular boundaries. The first is the equilibrium temperature attained by an isothermal layer subjected to radiation incident on both boundaries and with radiation being the only means of energy transfer. The balance of absorbed and emitted energy is

$$
\begin{aligned}
\int_{0}^{\infty}\left(q_{\lambda+1}+q_{\lambda, 2}\right) \alpha_{\lambda}\left(n, a_{\lambda} D\right) d \lambda & \\
& =2 \int_{0}^{\infty} \epsilon_{\lambda}\left(n, a_{\lambda} D\right) e_{\lambda b}\left(\lambda, T_{n}\right) d \lambda
\end{aligned}
$$

where $\epsilon_{\lambda}=\alpha_{\lambda}$. Equation (7) is solved for the equilibrium temperature $T_{w}$.

A sccond example is the radiative cooling of a layer initially at temperature $T_{0}$, when placed in surroundings at $T_{1}=T_{2}$ $=T_{\text {e. }}$ This is governed by the transient energy equation $-\rho c D \frac{\partial T_{w}}{\partial t}=2 \int_{0}^{\infty} \epsilon_{\lambda}\left(n, a_{\lambda} D\right)\left[e_{\lambda b}\left(\lambda, T_{w}\right)\right.$

$$
\left.-e_{\lambda b}\left(\lambda, T_{e}\right)\right] d \lambda
$$

For a spectrally banded calculation with $M$ bands with each band designated by $m$,

$$
-\rho c D \frac{\partial T_{w}}{\partial t}=2 \sum_{m=1}^{M} \epsilon_{m}\left(n, a_{m} D\right)\left[F_{m}\left(T_{w}\right) \sigma T_{w}^{4}-F_{m}\left(T_{e}\right) \sigma T_{e}^{4}\right]
$$

This is placed in dimensionless form and integrated to give

$$
I\left(\tilde{T}_{w}\right)=\frac{1}{2} \int_{T_{w^{\prime}}(n)}^{1} \frac{d \tilde{T}_{w}^{*}}{\sum_{m=1}^{M} \epsilon_{m}\left(n, a_{m} D\right)\left[F_{m}\left(\tilde{T}_{w}^{*} T_{0}\right) \tilde{T}_{w}^{* 4}-F_{m}\left(T_{e}\right) \tilde{T}_{e}^{4}\right]}
$$

Equation (10) is numerically integrated to yield cooling curves for various $T_{0}$ and $T_{e}$.

\section{Results and Discussion}

Absorptance of Layer. For specular boundaries the layer absorptance was evaluated from Eq.(1) for both diffuse and normal incidence of external radiation. Results are in Fig. 2(a) for refractive indices from 1 to 4 and optical thicknesses up to 4 . For $a_{\lambda} D$ larger than 4 almost all incident energy that is not reflected is absorbed in the layer. The layer absorptance then approaches an asymptotic value of one minus the external reflectivity of the boundary. Since the boundary reflectivity is smaller for normal incidence than for diffuse, the $\alpha_{\lambda_{s}}$ is larger for normal incidence. For $a_{\lambda} D<4$ the $\alpha_{\lambda ;}$ is influenced by the radiative path lengths within the layer. For a large $n$, incident diffuse energy is refracted into a small cone angle around the normal direction through the layer. Radiation within the nonscattering layer then travels along paths not much longer than the path length $D$ for normal incidence; hence, for large $n$ the $\alpha_{\lambda_{s}}$ becomes independent of normal or diffuse incidence. There are differences in $\alpha_{\lambda, s}$ when $n$ is near 1 and refraction is therefore small. Diffuse radiation then travels along longer paths and $\alpha_{\lambda, s}$ becomes increased. This is compensated somewhat by the reflectivity of the external boundary being larger for diffuse incident radiation than for radiation incident in the normal direction.

In Fig. $2(b)$ the absorptance of a layer with diffuse boundaries is compared with that for specular boundaries; the incident radiation is diffuse. The nature of the boundary has a very significant effect when $a_{\lambda} D$ is less than about 3 . For $n=$ 1 there are no surface reflections so the results are the same for the solid and dashed curves. As $n$ is increased the absorptance values become increasingly different, so for $n=4$ with $a_{\lambda} D<3$ there is a substantial difference in $\alpha_{\lambda}$. For specular boundaries $\alpha_{\lambda}$ decreases as the angle of refraction becomes smaller with larger $n$, thus providing shorter path lengths for absorption in the layer. For diffuse boundaries the increase in $\alpha_{\lambda}$ with $n$ is attributed to radiation within the layer being diffused at the boundaries into directions larger than the critical angle for reflection. This tends to retain energy within the layer and increase its absorption as radiation travels internally by multiple reflections. The effect that this can have on the layer temperature is shown by results that follow as calculated from Eqs. (7) and (10). If a layer with specular boundaries has internal scattering it will act more like a diffuse layer; the results here are without internal scattering and are expected to show the maximum possible effect of the two different types of boundaries.

Effect of Surface Condition on Layer Temperature. To illustrate how surface condition can affect the layer temper- 

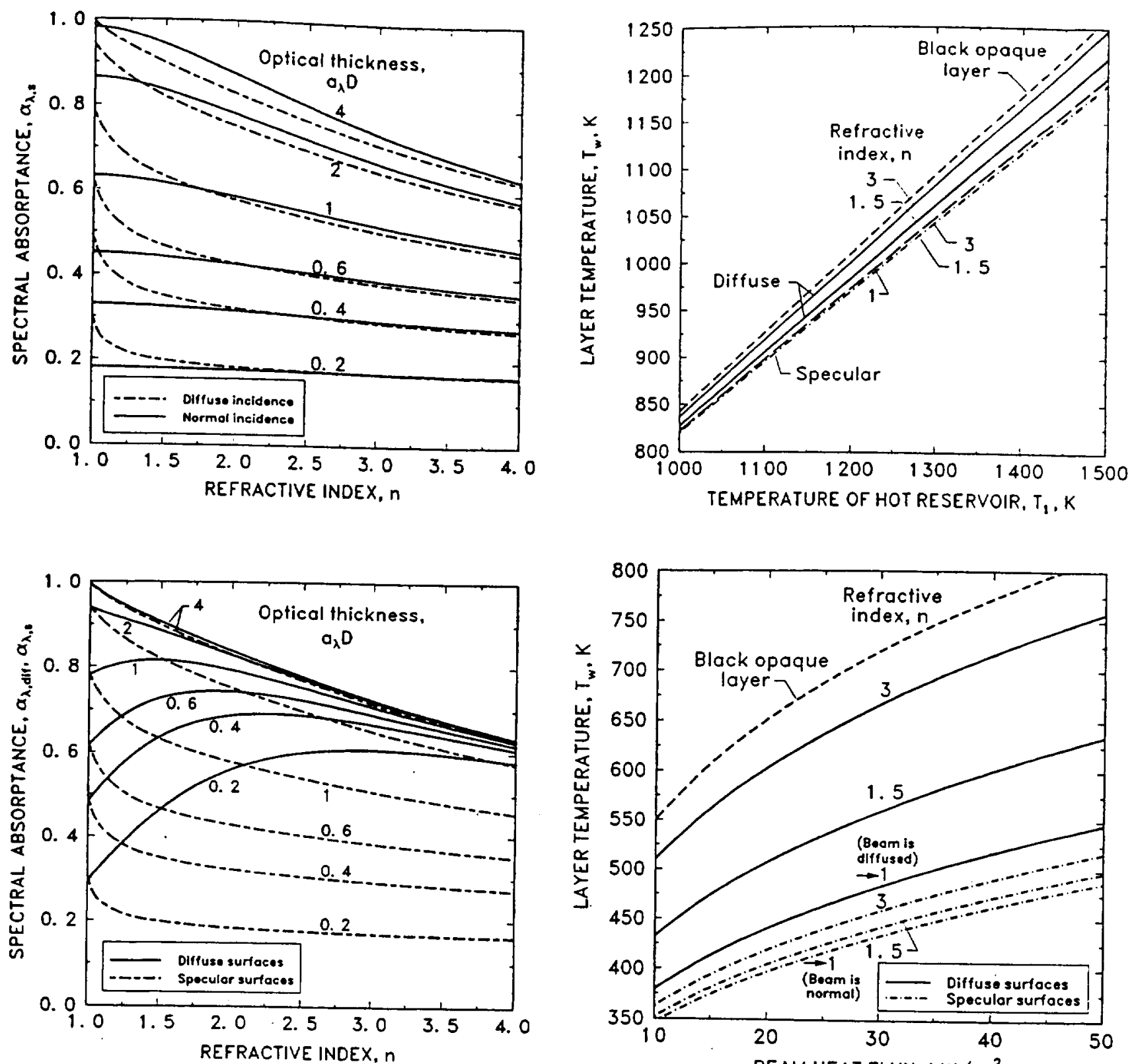

Fig. 2 The effect of diffuse or specular boundaries, and beam or diffuse Incidence, on spectral absorptance (or spectral emiltance) of a plane layer without internal scattering as a function of optical thickness and refractive index: (a) Boundaries are specular and incident external ra. diation is diffuse or is in the normal direction; (b) boundarles are diffuse, or are specular with diffuse incident radlation

ature, results are given for a layer with $D=0.5 \mathrm{~cm}$ and $a_{\lambda}$ approximated as constant in each of three spectral bands, similar to the properties of glass: $a_{\lambda}=0.2,4,100 \mathrm{~cm}^{-1}$ for $\lambda=0-2.7,2.7-4.4,4.4-\infty \mu \mathrm{m}$. Using these properties the spectral absorptance and emittance in each band was obtained from Eqs. (1) and (6). The equilibrium temperature $T_{w}$ for heat transfer only by radiation was then obtained from Eq. (7), and transient radiative cooling results were evaluated from Eq. (10).

Figures $3(a)$ and $3(b)$ show how equilibrium temperatures are affected for two different conditions of the incident radiation. As shown by Fig. $2(b)$, when $a_{\lambda} D$ is small the specular and diffuse boundary conditions produce significantly different $\alpha_{\lambda}$. Hence for a layer without internal scattering the condition of the boundary can produce large differences in energy absorption if there is large incident energy in wavelength regions where $a_{\lambda} D$ is small. For a high temperature radiant source

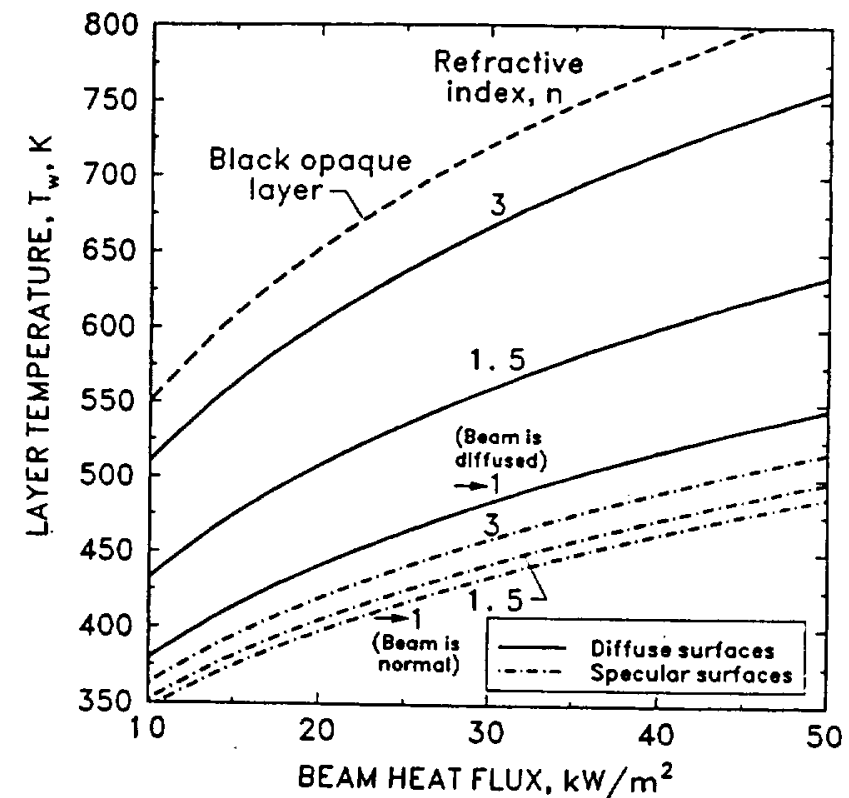

Fig. 3 Effect of diffuse or specular boundaries on the equillbrium tem. perature of a layer with a refractive index of 1.5 or 3 , and for the limiting case $n=1$. The spectral absorplion properties are similar to glass ( $a_{\lambda}$ $=0.2,4,100 \mathrm{~cm}^{-1}$ for $\left.\lambda=0-2.7,2.7-4.4,4.4-\infty \mu \mathrm{m}\right)$ and there is no Internal scattering; $T_{2}=300 \mathrm{~K}$. (a) Incident radiation on one side is from a hol reservoir at $T_{1}$. (b) Incident radiation on one side is a beam normal to the layer with a spectral distribution like that of solar radiation.

the incident energy fraction is large in the short wavelength region where semitransparent materials can have a small $a_{\lambda}$. The wavelength selective heat balance can then be sensitive to the specification of specular or diffuse boundary conditions. Reradiation is at longer wavelengths where $a_{\lambda} D$ is generally large; hence from the results in Fig. $2(b)$ the reradiated energy is not affected significantly by the boundary being specular or diffuse. For a moderate source temperature both the incident and emitted energy will be at long wavelengths where $a_{\lambda} D$ can be large causing $\alpha_{\lambda}$ to be insensitive to specular or diffuse boundaries.

In Fig. 3(a) the layer temperature $T_{w}$ is given for radiative heating by blackbody reservoirs with $T_{1}$ given on the abscissa, and $T_{2}=300 \mathrm{~K}$. The $T_{w}$ is given for diffuse and specular boundaries with the layer refractive index $n=1,1.5$, and 3 . For $n=1$ there are no surface reflections so the energy balance 


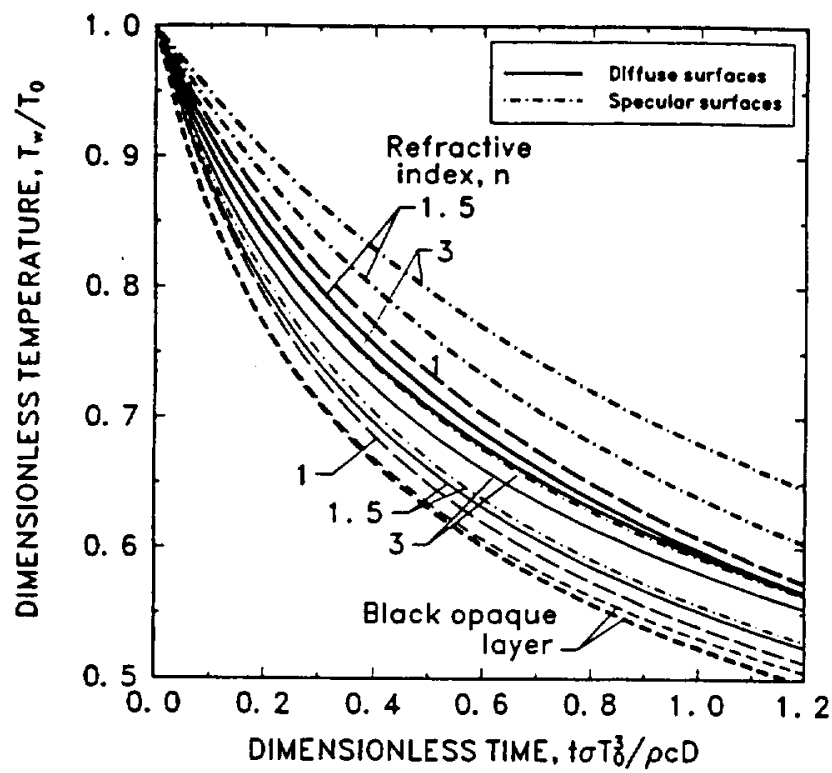

Fig. 4 Transient cooling of a tayer with initial temperature $T_{0}=1000$ $K$ (thin Ilnes) or $2000 \mathrm{~K}$ (thick llnes), $T_{0}=300 \mathrm{~K}$, and with the same absorption properties as for Flg. $3\left(a_{\lambda}=0.2,4,100 \mathrm{~cm}^{-1}\right.$ for $\lambda=0-2.7$,

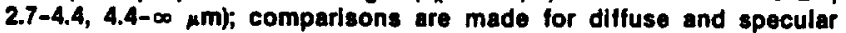
boundaries with $n=1.5$ and 3 (for $n=1$ there are no surface reflectlons)

depends only on internal absorption and emission. For $n>1$ the higher $\alpha_{\lambda}$ for small $a_{\lambda} D$ with diffuse boundaries produces increased absorption. The resulting $T_{w}$ for diffuse boundaries is somewhat higher than for the specular case and the difference increases to a maximum of about $50 \mathrm{~K}$ as $n$ and $T_{1}$ are increased. The temperature for an opaque black layer (or an opaque gray layer with diffuse boundaries) is shown as an upper limit. From Eq. (7) this is given by $T_{w}=\left[\left(T_{1}^{4}+T_{2}^{4}\right) / 2\right]^{1 / 4}$.

For Fig. $3(b)$ the nature of the incident radiation is changed on the hot side of the layer. The incident energy is a beam normal to the layer with its heat flux given on the abscissa. Two limiting cases are given for $n-1$; the solid curve is for the beam being diffused at the boundary, and the dot-dash curve is for the beam remaining normal to the boundary as it passes into the layer. The heat flux is high (for comparison the solar constant at earth orbit is about $\left.1.35 \mathrm{~kW} / \mathrm{m}^{2}\right)$ and has the same spectral distribution as solar energy (blackbody spectrum at $5780 \mathrm{~K}$ ). This spectrum provides a much larger energy fraction in the short wavelength region than the hot reservoir in Fig. $3(a)$ and produces substantially higher $T_{w}$ when the boundaries are diffuse. The upper limit is shown for a black opaque plate as given by $T_{w}=\left[\left(q_{\text {beam }} / \sigma+T_{2}^{4}\right) / 2\right]^{1 / 4}$.

Effect of Surface Condition on Transient Radiative Cooling. Transient radiative cooling results from $\mathrm{Eq} .(10)$ are in Fig. 4 for two initial temperatures $T_{0}=1000 \mathrm{~K}$ (thin lines) and $2000 \mathrm{~K}$ (thick lines), and $T_{e}=300 \mathrm{~K}$. The absorption properties are the same as for Fig. 3. For $n=1$ there are no surface reflections and hence no effect on cooling of the boundary being specular or diffuse. For $T_{0}=1000 \mathrm{~K}$ most of the energy radiated by the layer is in the long wavelength region where $a_{\lambda} D$ is large for the spectral properties used here. For large $a_{\lambda} D$, Fig. $2(b)$ shows that increasing $n$ decreases the $\alpha_{\lambda}$ $=\epsilon_{\lambda}$ so the cooling is less rapid. The $\epsilon_{\lambda}$ is smaller for specular surfaces, which produce longer cooling times. The situation changes somewhat when $T_{0}$ is increased $102000 \mathrm{~K}$. A greater fraction of the energy is then in the short wavelength regions where $a_{\lambda} D$ is small. This changes the trend for the solid curves (diffuse surfaces) in Fig. 2(b) which, for small $a_{\lambda} D$, show $\epsilon_{\lambda}$ increasing with $n$ to a maximum. This enhances the cooling rate with the increase in $n$ as shown in Fig. 4 by the thick solid curves as compared with the $n=1$ results (thick dashed curve).
The limiting results are also shown for cooling of a black opaque layer. In this instance there is no effect of spectral selectivity and the layer with highest initial temperature $T_{0}$ cools most rapidly.

\section{Conclusions}

Equilibrium temperatures of an absorbing-emitting layer were obtained for exposure to incident radiation and with the layer boundaries either specular or diffuse. For high refractive indices the surface condition can influence the radiative heat balance if the layer optical thickness is small. Hence for a spectrally varying absorption coefficient the layer temperature is affected if there is significant radiative energy in the spectral range with a small absorption coefficient. Similar behavior was obtained for transient radiative cooling of a layer where the results are affected by the initial temperature and hence the fraction of energy radiated in the short wavelength region where the absorption coefficient is small. The results are for a layer without internal scattering. If internal scattering is significant, the radiation reaching the internal surface of a boundary is diffused and the effect of the two different surface conditions would become small.

\section{References}

Gardon, R. 1956, "The Emissivity of Transparent Materials," Journal of the American Ceromic Saciety, Vol. 39, No. 8, pp. 278-287.

Richmond, J. C., 1963, "Relation of Emittance to Other Optical Properties," Journal of Research of the National Bureau of Siandards, Vol. 67C, No. 3, pp. 217-226.

Siegel, R., and Howell, J. R., 1992, Thermal Radiation Heat Transfer, 3rd ed., Hemisphere Publishing Corporation, Washington, DC

\section{Forced Convective Cooling of Optical Fiber During Drawing Process}

\section{S. Roy Choudhury, ${ }^{1}$ Y. Jaluria, ${ }^{1}$ T. Vaskop- oulos, ${ }^{1}$ and C. E. Polymeropoulos ${ }^{1}$}

\author{
Nomenclature \\ $h=$ surface convection heat transfer coefficient \\ $H=$ channel width $=r_{H}-r_{0}$ \\ $K=$ thermal conductivity \\ $m=$ fluid mass flow rate \\ $\mathrm{Nu}=$ local Nusselt number $=h r_{0} / K_{f}$ \\ $r, x=$ coordinate distances in the radial and axial \\ directions, respectively \\ $r_{0}=$ radius of the moving fiber \\ $R, X=$ dimensionless $r$ and $x, R=x / r_{0}, X=x / r_{0}$ \\ $T=$ local physical temperature
}

\footnotetext{
'Department of Mechanical and Aeruspace Engineering, Rutgers University, New Brunswick, NJ 08903.

Contributed by the Heat Transfer Division and presented at the National Heat Transfer Conference, Allanta, Georgia. August 8-11, 1993. Manuscript received by the Heat Transfer Division June 1993; revision recejved November 1993. Keywords: Conjugate Heat Transfer, Forced Convection, Materials Processing and Manufacturing Processes. Associate Technical Editor: R. Viskenta.
} 